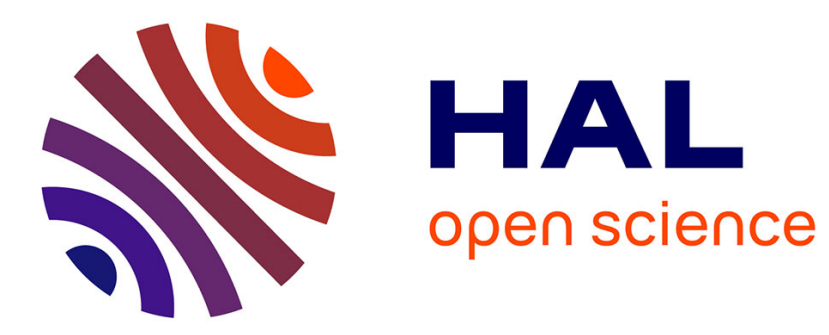

\title{
Conception and reproducibility study of efficient evanescent Raman converters based a nanofiber immersed in a liquid
}

Maha Bouhadida, Jean-Charles Beugnot, Philippe Delaye, Kien Phan Huy, Sylvie Lebrun

\section{To cite this version:}

Maha Bouhadida, Jean-Charles Beugnot, Philippe Delaye, Kien Phan Huy, Sylvie Lebrun. Conception and reproducibility study of efficient evanescent Raman converters based a nanofiber immersed in a liquid. SPIE Laser Resonators, Microresonators, and Beam Control, Feb 2020, San Francisco, United States. hal-03360771

\section{HAL Id: hal-03360771 \\ https://hal.science/hal-03360771}

Submitted on 1 Oct 2021

HAL is a multi-disciplinary open access archive for the deposit and dissemination of scientific research documents, whether they are published or not. The documents may come from teaching and research institutions in France or abroad, or from public or private research centers.
L'archive ouverte pluridisciplinaire $\mathbf{H A L}$, est destinée au dépôt et à la diffusion de documents scientifiques de niveau recherche, publiés ou non, émanant des établissements d'enseignement et de recherche français ou étrangers, des laboratoires publics ou privés. 


\title{
Conception and reproducibility study of efficient evanescent Raman converters based a nanofiber immersed in a liquid
}

\author{
Maha Bouhadida*a, Jean-Charles Beugnotb, Philippe Delayea, Kien Phan Huyb, Sylvie Lebruna \\ a Laboratoire Charles Fabry, Institut d'Optique, CNRS, Université Paris-Saclay, 91127 Palaiseau \\ cedex, France
}

b Institut FEMTO-ST, Université Bourgogne Franche-Comté, CNRS, 25030 Besançon, France

\begin{abstract}
We demonstrate, build and optimize evanescent Raman converters at the sub-nanosecond regime based on a silica nanofiber immersed in ethanol. Two different standard silica fibers (SMF28, 460HP) are tested and compared. The converters are pumped at $532 \mathrm{~nm}$ and deliver pulses at $630 \mathrm{~nm}$, which is the first Stokes order wavelength of ethanol. They present highly reproductible performances. A maximum output Stokes energy of $0.29 \mu \mathrm{J}$ is usually reached with an external conversion efficiency of $60 \%$. Lowering the Raman threshold and pushing up the nanofiber breakdown allow a higher conversion operating range, that is the conception key of these converters.
\end{abstract}

Keywords: nanofiber, evanescent nonlinearity, Raman scattering

\section{INTRODUCTION}

Optical nanofibers are fibers stretched until reaching diameters comparable or smaller than the light wavelength. For these diameters, nanofibers exhibit a strong confinement of light enabling the investigation of nonlinear effects [1] like the generation of supercontinuum [2]. They also have an intense evanescent field that can be exploited for different applications such as optical traps [3], optical sensing [4] and spectroscopy [5]. However, the generation of nonlinear effects in the evanescent field of the nanofiber remains limited. In this paper, we are interested in the Raman scattering in the evanescent field of a nanofiber immersed in a liquid as the "evanescent nonlinearity". The evanescent Raman effect was previously observed with a few-cm-length nanofiber immersed in ethanol and in toluene by using sub-nanosecond pump pulses at $532 \mathrm{~nm}$. This first observation was investigated using nanofibers that allow a maximal modal Raman gain [6]. The output energy of the first Stokes order of the Raman liquid was limited to $0.11 \mu \mathrm{J}$ by the breakdown of the nanofiber and never exceeded the transmitted pump energy. Extracting more Stokes energy requires increasing the Raman conversion operating range. It is then mandatory to decrease the Raman threshold and to increase the nanofiber breakdown threshold. One alternative is to decrease the pump pulse laser duration until a few tens of ps. Another alternative is to keep the subnanosecond regime and to increase the diameter of the nanofiber. However, higher diameters would increase the Raman threshold because the intensity of the evanescent field would be decreased. A compromise has to be established by finding the nanofiber diameter that enables the extraction of the maximal output energy at the Stokes wavelength. In this context, we propose a design of a Raman converter based on a nanofiber immersed in a liquid that allows to extract the maximum of the Stokes energy [7]. Two different fibers are compared. The efficiency and the reproducibility of this evanescent Raman conversion are studied.

The paper is structured as follows: we dedicate the first section to model the evanescent Raman conversion. The structure of the converter is described in the second section. In the following section, we detail the nonlinear characterization of the proposed converter and we study the efficiency and the reproducibility of its conversion in the last section. We obtain an output Stokes energy of $0.29 \mu \mathrm{J}$ at $630 \mathrm{~nm}$ i.e. a value that is almost three times higher than the previous results. An external Raman conversion efficiency around $60 \%$ is reached with a high reproducibility opening the way to a new family of low-cost compact and efficient all fibered Raman sources that can be directly inserted in optical fibered networks. 


\section{EVANESCENT RAMAN CONVERSION MODELLING}

The nanofiber is composed of a waist and two symmetrical tapers which are the transition sections between this waist and the unstreched parts of the fiber $\mathrm{L}_{1}$ and $\mathrm{L}_{2}$ as shown in Figure 1. The input pump beam is injected in $\mathrm{L}_{1}$ and propagates in the core of the fiber. It then enters the left taper where the fiber dimensions (core radius $r_{c o}$, cladding radius $\left.r_{c l a d}\right)$ start to be progressively reduced. The taper is modeled as a three-layer structure combining the fiber core, the fiber cladding and the surrounding external medium. The pump beam enters the nanofiber waist which is assimilated to a two-layer structure composed by the fiber cladding and the external medium since the fiber core can be totally neglected in this part. The modes are considered to be scalar in the taper. For the nanofiber's radii equal or lower than the wavelength, the propagating modes are assumed to be vectorial because of the large index. The beam then propagates in the right taper and is collected at the end of the un-stretched part $\mathrm{L}_{2}$ as core mode. By immersing the nanofiber in a liquid, the incident pump photons of the evanescent field are scattered through Raman diffusion under the form of lower energy photons called Stokes photons. That is what we call "evanescent Raman conversion" [7,8]. For example, if the pump pulses are emitted at $\lambda_{\mathrm{p}}=532 \mathrm{~nm}$ and the immersing liquid is pure ethanol, the first Stokes order photons are scattered at $\lambda_{\mathrm{s}}=630 \mathrm{~nm}$. The evanescent part of the mode propagating in the external medium is significant. Figure 2 shows the fraction of the evanescent part of the fundamental mode field $f_{e v}$ as a function of the nanofiber radius for $\lambda_{\mathrm{p}}=532 \mathrm{~nm}$ and pure ethanol whose expression is the following:

$$
f_{e v}=\frac{\iint_{\text {evanescent area }}(e \times h) \cdot \hat{z} d A}{\iint_{\text {total area }}(e \times h) \cdot \hat{z} d A}
$$

Where $e$ and $h$ are the respectively electric and the magnetic fields. For the nanofiber's radii range we are considering (between $220 \mathrm{~nm}$ and $300 \mathrm{~nm}$ ) and for the fundamental mode HE11, $f_{e v}$ is always higher than 0.38 .

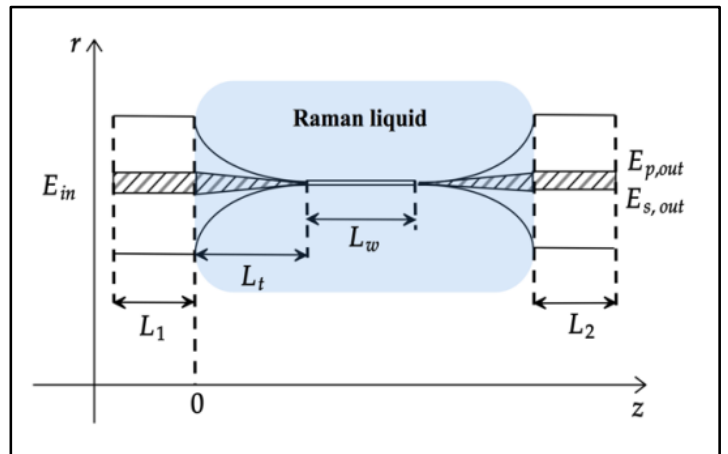

Figure 1. Evanescent Raman conversion scheme.

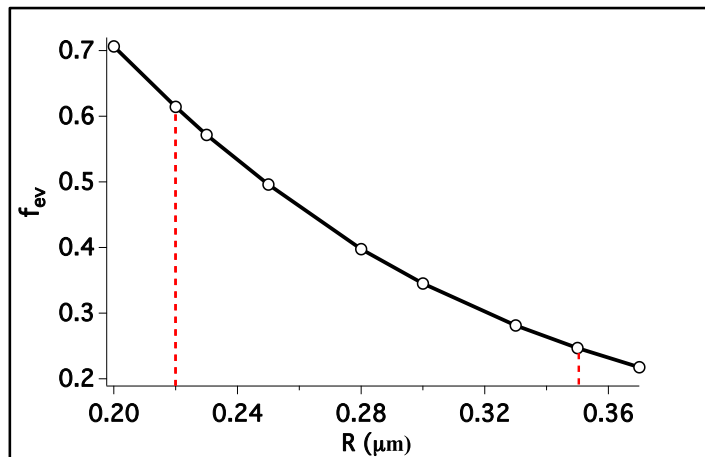

Figure 2. Evanescent fraction of the pump field on $\mathrm{HE} 11$ at $532 \mathrm{~nm}$ versus the nanofiber radius.

One key parameter behind the evanescent Raman conversion is the evanescent Raman threshold. We consider that the

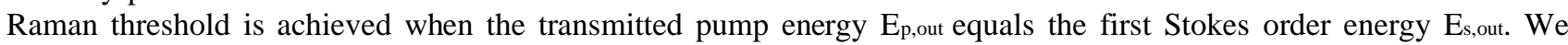
introduce the critical parameter $\gamma$ expressed as $\gamma=g_{R m} L_{w} P_{i n}$ where $g_{R m}$ is the modal Raman gain (in m-1.W-1) and $P_{\text {in }}$ is the peak pump power [9]. For sub-nanosecond pulses, $\gamma \approx 23$ at the Raman threshold [6, 7]. The expression of the modal Raman gain $g_{R m}$ is the following [10]:

$$
g_{R m}=\frac{\varepsilon_{0} c^{2} \iint_{\text {active area }} g_{L} n_{L}^{2}\left|e_{p} \cdot e_{s}\right|^{2} d A}{\iint_{\text {total area }}\left(e_{p} \times h_{p}\right) \cdot \hat{z} d A \iint_{\text {total area }}\left(e_{s} \times h_{s}\right) \cdot \hat{z} d A}
$$


where $g_{L}$ (in $\mathrm{m} . \mathrm{W}_{-1}$ ) is the Raman gain coefficient of the liquid and $\mathrm{n}_{L}$ is its refractive index. $\epsilon_{0}$ and $c$ are, respectively, the dielectric permittivity and the light celerity in vacuum; $e$ and $h$ represent respectively the electric and the magnetic fields; $\mathrm{p}$ and $\mathrm{s}$ stand for, respectively, pump and Stokes.

Since the modal Raman gain of the crossed interactions between modes is very weak, we assume that both the pump and the Stokes beams are propagating on the same mode.

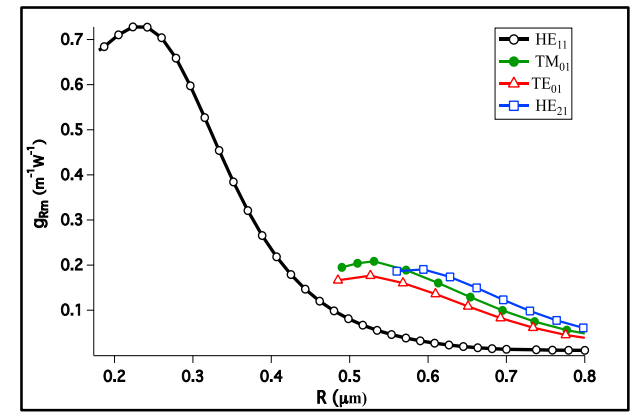

Figure 3. $g_{R m}$ versus nanofiber radius for HE11, TM01, TE01 and HE21.

The variations of $g_{R m}$ with the nanofiber radius for the modes HE $11, \mathrm{TM} 01, \mathrm{TE} 01$ and $\mathrm{HE}_{21}$ are shown in Figure 3 . We observe that there is a radius optimizing $g_{R m}$ for each plot. When the radius is smaller, the beam structure approaches a plane wave spreading along important distances and so, the amplitude of the evanescent field decreases reducing the gain. However, larger radii make the mode more confined in the fiber core and so, the Raman gain decreases. For nanofiber radii between $220 \mathrm{~nm}$ and $350 \mathrm{~nm}$ and an input peak power of $1 \mathrm{~kW}$, the evanescent Raman threshold can be achieved with waist lengths $L_{w}$ between $5 \mathrm{~cm}$ and $8 \mathrm{~cm}$ which is possible with our pulling system. For the higher order modes, higher radii are needed to achieve the maximal modal Raman gain. This gain does not exceed $0.2 \mathrm{~m}_{-1}$. $\mathrm{W}_{-1}$ but it can be compensated by pulling longer nanofibers ( $L_{w}=15 \mathrm{~cm}$ for TM01 and at $R=508 \mathrm{~nm}$ ).

\section{EVANESCENT RAMAN CONVERTER STRUCTURE}

The proposed evanescent Raman converter is based on the component drawn in the section above and depicted in Figure 1. The immersed parts in the Raman liquid are the nanofiber waist and the tapers. $L_{1}$ and $L_{2}$ are needed to make the manipulation easier and to facilitate the light injection and collection. $L_{1}$ and $L_{2}$ should be long enough for practical use but short enough to avoid parasitic nonlinear effects as it will be explained later.

The nanofiber is fabricated following the "pull and brush" technique with the pulling system described in [6]. A butane flame softens the central part of the fiber while two translation stages elongate it. The flame flow and intensity and the stages are fully computer-controlled, which allows to create reproducible nanofibers with high light transmission. Based on the target parameters of the nanofiber (nanofiber radius $R$, waist length $L_{w}$ ), the pulling process is divided into $N$ cycles. During each cycle, the radius of the fiber cladding is reduced by a constant ratio to reach the nanofiber waist radius at the end of the last cycle and the taper is elongated. At the $\mathrm{n}^{\text {th }}$ cycle, the heat zone length is chosen as a power law of $\mathrm{n}$, enabling to create few-cm length tapers. This fabrication process is performed in a class-5 cleanroom to avoid the presence on the dust. As soon as the nanofiber is immersed in the Raman liquid, the experiment can be carried on in laboratory conditions. Based on the values of $g_{R m}$ represented in Figure 3, the converter's radii are chosen to be between $220 \mathrm{~nm}$ and $350 \mathrm{~nm}$, which corresponds to a modal Raman gain between $0.35 \mathrm{~m}_{-1}$. W-1 and $0.98 \mathrm{~m}_{-1}$. $\mathrm{W}_{-1}$. $\mathrm{L}_{\mathrm{w}}$ is fixed to $8 \mathrm{~cm}$ which is the highest length we can have with our pulling system.

We choose to pull the nanofiber from two different fibers : a single mode fiber at $532 \mathrm{~nm}$ (460HP from Thorlabs) and a single mode fiber at $1550 \mathrm{~nm}$ (SMF28 from Corning). Since the SMF28 fiber is highly multimode at $\lambda_{\mathrm{p}}=532 \mathrm{~nm}$, high order modes should be filtered to allow only the propagation of the fundamental mode HE 11 . One way to filter higher order modes is to pull $\mathrm{L}_{1}$ over a few-cm length until reaching the cutoff frequency of the first higher order mode and to keep the fiber short and straight after the filter. 
To guarantee high light transmission, special attention should be paid to the tapers adiabaticity. The tapering angle has to be small enough to avoid coupling between the fundamental and higher order modes. We study the adiabaticity for the fundamental mode $\mathrm{LP}_{01}$. The adiabaticity criterion that is usually adopted is [11]:

$$
\frac{1}{Z_{12}} \geq \frac{1}{r} \frac{d r}{d z}
$$

$\mathrm{r}(\mathrm{z})$ is the local radius of the taper transitions, $\frac{1}{r} \frac{d r}{d z}$ is the normalized taper slope, $Z_{12}=\frac{2 \pi}{\beta_{1}-\beta_{2}}$ is the beating length. $\beta_{1}$ is the local propagation constant of the pump mode LP01 and $\beta_{2}$ is the local propagation constant of the mode having the highest chance to couple with it because of the azimuthal dependence, i.e. LP02.

Fixing $L_{w}$ to $8 \mathrm{~cm}$, we consider three nanofiber samples : NF1 $(R=220 \mathrm{~nm}), \mathrm{NF} 2(\mathrm{R}=300 \mathrm{~nm})$ and NF3 $(\mathrm{R}=350 \mathrm{~nm})$. The tapers created for these three samples are the same for both fibers SMF28 and 460HP with lengths Lt respectively equal to $5 \mathrm{~cm}, 4.5 \mathrm{~cm}$ and $4 \mathrm{~cm}$. The normalized slopes of the three tapers profiles are plotted as a function of the relative radius in Figure 4 . The adiabaticity boundaries at $532 \mathrm{~nm}$ and $630 \mathrm{~nm}$ for both fibers are also represented.

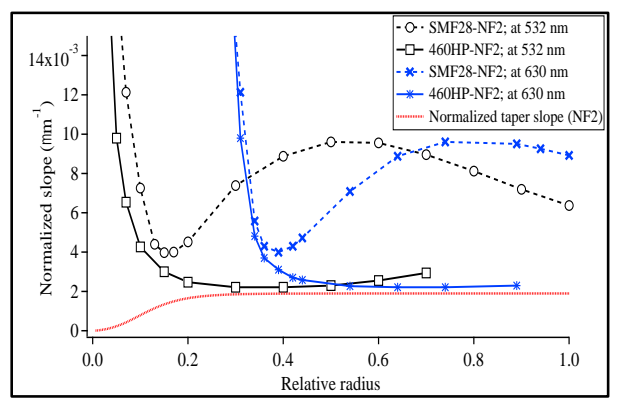

Figure 4. Tapers immersed in ethanol at $532 \mathrm{~nm}$ for the SMF28 (empty round) and 460HP (empty square). Same at 630 $\mathrm{nm}$ for the SMF28 (cross) and 460HP (star).

Figure 4 shows that making the taper adiabatic with SMF28 is easier than with 460HP for the fabricated taper profiles. Indeed, the adiabaticity boundary for the SMF28 taper curve has a punctual minimum of $0.0035 \mu \mathrm{m}-1$ for a relative radius of 0.15 . This minimum is not as punctual for the $460 \mathrm{HP}$ taper and it is maintained over a relative radius range extending from 0.3 to 0.55 keeping a value of $0.0019 \mu \mathrm{m}-1$. When the nanofiber is immersed in ethanol, the light transmission of the whole component at $532 \mathrm{~nm}$ defined as the ratio between the output energy $E_{p, o u t}$ and the input energy $E_{\text {in }}$ is experimentally $90 \%$ for the SMF28 and $87 \%$ for the $460 \mathrm{HP}$. These transmission values are almost the same for the three taper profiles. The adiabatic boundary curves are right-shifted when the wavelength increases which keeps the tapers adiabatic at the Stokes wavelength of ethanol $\lambda \mathrm{s}=630 \mathrm{~nm}$

\section{NONLINEAR CHARACTERIZATION OF THE CONVERTER}

In order to obtain the maximal Raman conversion efficiency, controlling the undesired nonlinear effects is mandatory. The experimental setup presented in Figure 5.

A pulsed pump laser (from HORUS) emits a beam at $532 \mathrm{~nm}$ with a maximum available peak pump power of $7 \mathrm{~kW}$, a pulse duration of $900 \mathrm{ps}$ and a frequency rate repetition of $4.7 \mathrm{kHz}$. A lens L1 with a focal distance of $10 \mathrm{~cm}$ collimates the pump light and the association of a half plate and a polarizing beam splitter helps us to control the incident pump power since the laser is linearly polarized. Part of the pump beam then passes through a glass plate. The converter is immersed in a tank filled with the Raman liquid. The pump beam is injected by a microscope objective (x20, NA 0.35 ) in the untapered input end of the fiber $\mathrm{L}_{1}$, propagates in the nanofiber and is collected at the output end of the fiber $\mathrm{L}_{2}$. The role of the glass plate is to reflect the backward beams into an optical spectrum analyzer OSA2 while OSA1 will analyze the forward beams. 


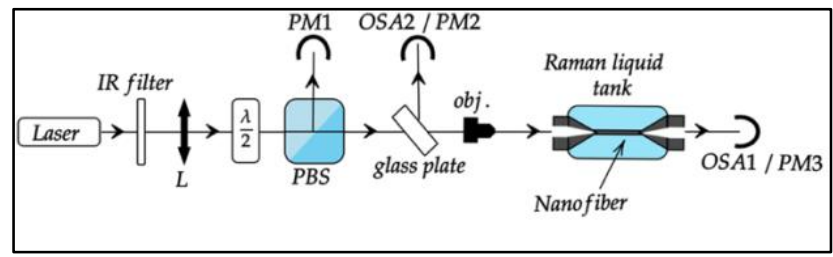

Figure 5. Experimental setup scheme for the nonlinear characterization of evanescent Raman converter.

We depict in Figure 6 the spectra of the forward beam collected from the three considered nanofiber radii when the immersing liquid is ethanol.

We observe that there is no forward silica Raman scattering for the whole converter at $545 \mathrm{~nm}$. This is explained by the appropriate choice of $\mathrm{L}_{1}$ and $\mathrm{L}_{2}$ lengths (respectively $30 \mathrm{~cm}$ and $40 \mathrm{~cm}$ ) that are short enough and the silica Raman nanofiber threshold energy that is higher than the evanescent Raman threshold energy in ethanol.

No significant spectral broadening of the pump line is observed showing the absence of Kerr effect in silica and in ethanol [12].

In the sub-nanosecond regime, as the effective length of interaction and the pump length are of the same magnitude ( $\sim 10 \mathrm{~cm}$ ), backward nonlinear effects can be favored [13]. On OSA2, we observed backward Raman scattering, neither in silica nor in ethanol. We measured the backward energy at $532 \mathrm{~nm}$ versus the incident pump energy. The plots are almost linear for the three nanofibers, showing that there is not Brillouin scattering. The backward energy is due to parasitical reflections.

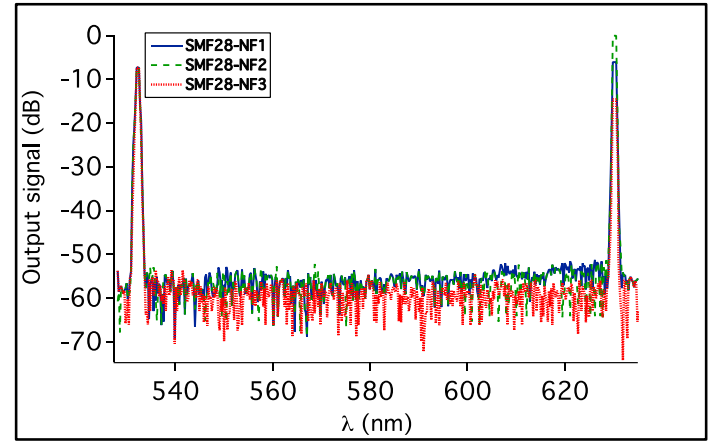

Figure 6. Collected forward spectra from OSA1 for nanofibers immersed in ethanol just before they break.

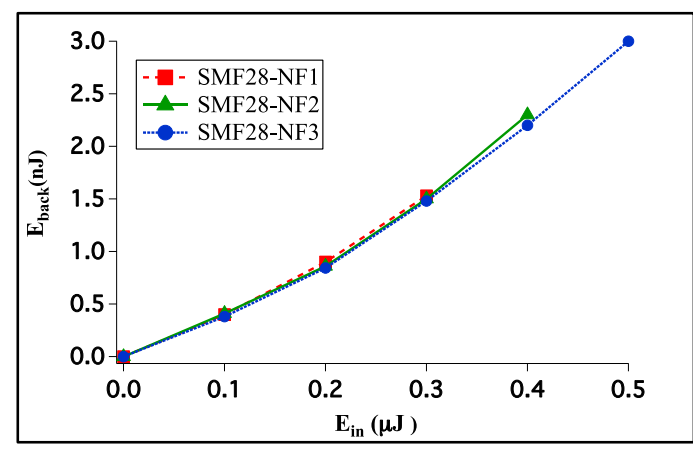

Figure 7. Backward energy versus the incident pump energy for the three studied nanofibers.

\section{CONVERSION EFFICIENCY AND REPRODUCIBILITY STUDIES}

\subsection{Conversion efficiency study of the converter}

Ensuring high modal Raman gains $g_{R m}$ is not sufficient to guarantee a high conversion efficiency of the evanescent Raman converter. The two key parameters that influence the conversion efficiency of the evanescent Raman converter are the Raman threshold energy $E_{R}$, th and the nanofiber optical breakdown energy $E_{b r e a k}$ [7]. We define the gap between the two parameters by "the Raman conversion operating range" $\Delta E$. The higher $\Delta E$ is, the more efficient the converter becomes [7].

We estimate in Table 1 the optical breakdown energies of the nanofiber samples NF1, NF2 and NF3 for both fibers. We present the results for two different external mediums (air and ethanol). Almost the same values are found with for both fibers. In air, the optical breakdown energy increases with the radius. In ethanol, the optical breakdown energy becomes higher for the three radii.

\begin{tabular}{|l|c|c|l|l|l|l|}
\hline Nanofiber & SMF28-NF1 & 460HP-NF1 & SMF28-NF2 & 460HP-NF2 & SMF28-NF3 & 460HP-NF3 \\
\hline Air $(\mathrm{n}=1)$ & 0.23 & 0.22 & 0.4 & 0.41 & 0.57 & 0.55 \\
\hline Ethanol $(\mathrm{n}=1.36)$ & 0.27 & 0.26 & 0.45 & 0.45 & 0.63 & 0.62 \\
\hline
\end{tabular}

Table 1. Optical breakdown energy in $\mu \mathrm{J}$ for different nanofibers diameters in different external mediums. 
We focus on the three SMF28 nanofibers. We compare the experimental and the theoretical Raman threshold energies. The theoretical Raman threshold energies are deduced from the expression of $\gamma$. The experimental and the theoretical Raman thresholds for NF1 and NF2 are in a good agreement and confirm the value of the critical parameter $\gamma \approx 23$ obtained in $[6,7]$. We were not able to reach the Raman threshold with NF3. A longer nanofiber would be needed. Theoretical and experimental Raman conversion operating ranges $\Delta E$ are represented in Table 2.

\begin{tabular}{|l|c|c|}
\hline Nanofiber & Experimental value & Theoretical value \\
\hline SMF28-NF1 & 0.02 & 0.025 \\
\hline SMF28-NF2 & 0.08 & 0.1 \\
\hline SMF28-NF3 & 0.005 & 0.007 \\
\hline
\end{tabular}

Table 2. Raman conversion operating ranges in $\mu \mathrm{J}$ for the tested nanofibers.

Based on the estimated Raman conversion operating ranges $\Delta E$, the evanescent Raman converter created with NF2 is expected to be more efficient than the one fabricated with NF1. We present in Figure 8 the transmitted pump energy $E_{p, o u t}$ and the output Stokes energy $E_{S, \text { out }}$ as a function of the incident pump energy $E_{\text {in }}$ for the three considered nanofibers (SMF28-NF1, SMF28-NF2, SMF28-NF3).

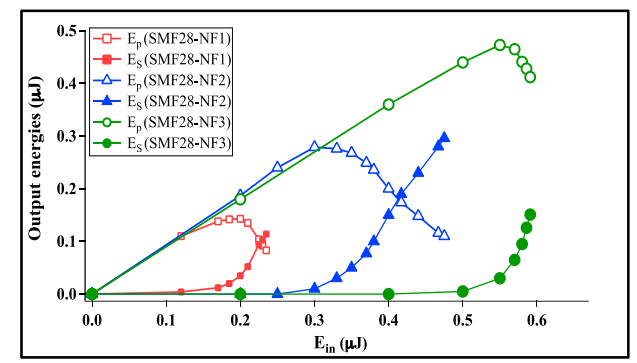

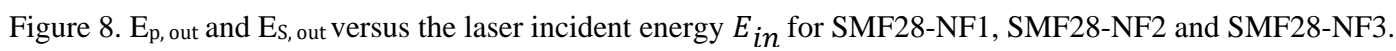

We define the external efficiency of the Raman conversion $\eta_{\text {ex }}$ as the fraction between the maximum output Stokes energy and the incident pump energy after reaching the Raman threshold. As expected, the highest Stokes energy was obtained with SMF28-NF2 and $\eta_{\mathrm{ex}}=60 \%$. An energy of $0.29 \mu \mathrm{J}$ at $630 \mathrm{~nm}$ is obtained just before the SMF28- NF2 broke. The Raman threshold is reached at $E_{i n}=0.41 \mu \mathrm{J}$. For SMF28-NF1, the maximum extracted Stokes energy is only $0.13 \mu \mathrm{J}$ and $\eta_{\text {ex }}$ is $51 \%$. With SMF28-NF3, a Stokes energy of only $0.15 \mu \mathrm{J}$ for an incident energy $E_{\text {in }}=0.58 \mu \mathrm{J}$ is achieved and the Raman threshold is not reached.

\subsection{Reproducibility study of the converter}

In order to study the reproducibility of the converter, we draw 20 samples of each nanofiber (SMF28-NF1, SMF28-NF2, SMF28-NF3). Figure 9 represents ER,th, Ebreak and Esmax for the 20 samples of SMF28-NF2. The results of SMF28-NF1 and SMF28-NF3 show the same trends.

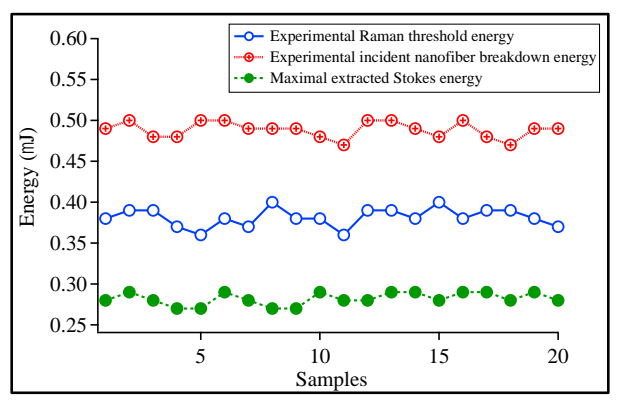

Figure 9. Experimental Raman threshold energies, incident nanofiber breakdown energies and maximal Stokes energies for the 20 samples of SMF28-NF2. 
We also compute the average and the standard deviation of $E_{S m a x}, E_{R, t h}, E_{b r e a k}$ for the 20 samples of SMF28-NF2 just before the breakdown in Table 3 .

\begin{tabular}{|l|l|l|}
\hline & Average $(\mu \mathrm{J})$ & Standard deviation $(\mu \mathrm{J})$ \\
\hline$E_{S \max }$ & 0.283 & 0.007 \\
\hline$E_{R, \text { th }}$ & 0.376 & 0.009 \\
\hline$E_{\text {break }}$ & 0.487 & 0.008 \\
\hline
\end{tabular}

Table 3. Average and standard deviation of the different energies computed for the 20 samples of SMF28-NF2.

\section{CONCLUSION}

We have proposed evanescent Raman converters in the sub-nanosecond regime based on a silica nanofiber immersed in ethanol. Two different standard silica fibers were tested and compared (SMF28 and 460HP). We obtained a maximum output Stokes energy of $0.29 \mu \mathrm{J}$ with an external conversion efficiency from the pump to the first Stokes order of ethanol of $60 \%$. One key parameter to optimize the extracted Stokes energy is the Raman conversion operating range, which is the gap between the Raman threshold energy and the optical breakdown energy. Indeed, the optical breakdown energy is estimated for different nanofiber diameters and for different external mediums. The Raman conversion operating range is a guideline for the conception of other evanescent Raman converters based on other liquids and/or fibers. The performances of the evanescent Raman converters are highly reproducible and other perspectives can be studied like the deposition of nonlinear polymers on the nanofiber surface opening the way to a new family of robust all-fibered components that can be directly inserted in optical networks without additional losses.

\section{REFERENCES}

[1] M.A. Foster, A.C. Turner, M. Lipson, A.L. Gaeta, Opt. Express 16(2), 1300 (2008).

[2] T.A. Birks, W.J. Wadsworth, P. St, J. Russell Opt. Lett. 25(19), 1415-1417 (2000).

[3] B. Gouraud, D. Maxein, A. Nicolas, O. Morin, J. Laurat, Phys. Rev. Lett. 114, 180503 (2015).

[4] J. Lou, Y. Wang, L. Tong, Sensors 14(4), 5823-5844 (2014).

[5] R. Garcia-Fernandez, W. Alt, F. Bruse, C. Dan, K. Karapetyan, O. Rehband, A. Stiebeiner, U. Wiedemann, D.

Meschede, A. Rauschenbeutel, Appl. Phys. B 105, 3-15 (2011).

[6] L. Shan, G. Pauliat, G. Vienne, L. Tong, S. Lebrun, Appl. Phys. Lett. 102, 201110 (2013).

[7] Bouhadida, M., Beugnot, JC., Delaye, P. et al. Appl. Phys. B (2019) 125: 228.

[8] L. Shan, G. Pauliat, G. Vienne, L. Tong, S. Lebrun, J. Eur. Opt. Soc. Rap. Publ. 8, 13030 (2013).

[9] R.G. Smith, Appl. Opt. 11, 2489-2494 (1972).

[10] M.D. Turner, T.M. Monro, S.V. Afshar, Opt. Express 17(14), 11565-11581 (2009).

[11] J.D. Love, W.M. Henry, W.J. Stewart, R.J. Black, S. Lacroix, F. Gonthier, IEE Proc. J. 138(5), 343-354 (1991).

[12] M.C. PhanHuy, P. Delaye, G. Pauliat, N. Dubreuil, F. Gérôme, B. Debord, F. Benabid, S. Lebrun, J. Eur. Opt. Soc. Rap. Publ. 13, 31 (2017).

[13] G. Fanjoux, J. Chrétien, A. Godet, K. Phan-Huy, JC. Beugnot, T. Sylvestre, Opt. Express 27, 29460-29470 (2019). 